\title{
MORAL BY DEFAULT? THE DYNAMIC TRADEOFFS BETWEEN HONESTY AND SELF-INTEREST
}

\author{
Haiyan $\mathrm{Wu}^{1 *}$, Siqi $\mathrm{CaO}^{23}$, Changlin $\mathrm{Bai}^{4}$, Kun Chen', Dean Mobbs ${ }^{\text {** }}$ \\ 1. Centre for Cognitive and Brain Sciences and Department of Psychology, \\ University of Macau, Macau, China, 999078
}

2. CAS Key Laboratory of Behavioral Science, Institute of Psychology, Beijing, China

3. Department of Psychology, University of Chinese Academy of Sciences, Beijing, China

4. School of Psychology, Beijing Normal University

5. Department of Humanities and Social Sciences and California Institute of Technology

Corresponding author:

HaiyanWu (hayanwu@,um.edu.mo),

Centre for Cognitive and Brain Sciences and Department of Psychology, University of Macau, Macau, China, 999078

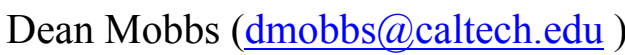

California Institute of Technology

Pasadena, California 91125, MC 228-77

Phone +16267148913

Email: dmobbs@caltech.edu

URL: www.deanmobbslab.com 


\begin{abstract}
Humans are often faced with social dilemmas that pit self-interest against honesty, yet questions arise about whether this trade-off reflects spontaneous default response or active suppression of the alternative response. Here, we created two message-sending tasks where decisions involved honesty as truth-telling or dishonesty as untruthful-message-sending. In our pre-registered study, participants joined the message task as the sender, who can send truthful or untruthful messages to earn more money for themselves, thus manipulating the conflict between self-interest and honesty. In experiment 1, we used mouse tracking (MT) to gain insight from movement trajectories and reaction time (RTs) of a forced-choice task with one fair default option. We found that being honest under conflict situations took longer for individuals who had higher self-interest concerns. In experiment 2 with a two-alternative forced-choice design without a default fair option on another sample to test whether selfinterest-seeking people would show a similar tendency to lie and how these behavioral patterns correlated with utility and drift-diffusion model (DDM) modeling parameters. We found lower RTs for untruthful responses when self-interest concern was higher. In both experiments, the mixed model of RTs indicated the interest-temptation (i.e., conflict) condition led to a longer RT for truth-telling. These results were also supported by a DDM showing a larger drift rate for untruthful, compared to truthful, responses in conflict trials. Overall, our findings further support the individual difference in moral default, which can be manifested in behavioral indices in decisions.
\end{abstract}

Keywords:

Dishonesty, Self-interest, Mouse-tracking, Utility model, Drift-diffusion model, Decisionmaking 


\section{Introduction}

Following the 2020 Presidential Election in the US, many Republicans, led by Donald Trump, vocally condemned the results stating that the election was stolen from them. Known as 'The Big Lie', ballot recounts and supreme court hearings rejected any question of foul play. However, lack of evidence and billion-dollar lawsuits have not dampened Trump and colleagues from continuing to openly question the election results. This persistent lie, however, underlies a mendacious strategy where hundreds of millions of dollars have been raised for the former president. Like other real-world examples, such as the malicious 'for profit' lies of Big Tobacco, scientific examples show that decisions that pit self-interest against honesty can result in cheating and misinformation (S. P. H. Speer, Smidts, \& Boksem, 2020).

An unanswered question concerns the psychological mechanisms by which such conscious and malevolent lies are propagated. The "Will" or "Grace" hypotheses of honesty puts forward opposite views on moral behavior: the 'Will Hypothesis' assumes that honesty requires cognitive control to suppress temptation to cheat (i.e., Default Selfish Hypothesis), while the 'Grace Hypothesis' assumes that people are intuitively moral and require cognitive control to dishonest behavior (J. D. Greene \& J. M. Paxton, 2009). Empirical research has confirmed that dishonesty requires more self-control resources than truthful responses (Vrij, Granhag, Mann, \& Leal, 2011). Therefore, previous research has theorized that honest responses are the default response (i.e., Truth-Default Theory) (Levine \& Schweitzer, 2014). However, a longer $\mathrm{RT}$ is reported for lying from a meta-analysis (Suchotzki, Verschuere, Van Bockstaele, BenShakhar, \& Crombez, 2017), indicating a cognitive cost of lying (Vrij, Fisher, Mann, \& Leal, 2006).

In self-serving contexts, self-interested individuals show more spontaneous dishonesty (Capraro, 2017; Shalvi, Eldar, \& Bereby-Meyer, 2012). For example, the monetary interest promotes dishonest response and deliberative honesty (Joshua D. Greene \& Joseph M. Paxton, 2009; Xu \& Ma, 2015). Shalvi et al. (2012) have found when humans are faced with temptations, they have an innate predisposition to lie for self-serving reasons. Such contrary perspective lead to an opposite assumption about whether an honest response is more effortful than a dishonest response in a self-interest context (Ariely \& Jones, 2012; Bereby-Meyer \& Shalvi, 2015). 
In this study, we also aimed to explore this controversial topic: whether people demonstrate spontaneous honesty or effortless dishonesty under a self-serving temptation context. Traditional RT-based studies provide evidence for the intuitive honesty hypothesis, showing longer RTs for untruthful responses (Suchotzki et al., 2017). Dishonesty involving both cognitive dynamics and sequential decision-making, and RTs do not allow us to know the automatic response during the initiation of the response (Walczyk, Harris, Duck, \& Mulay, 2014). We are still unclear whether there is a hesitation as an initial activation of a truthful response. To address the limitation of traditional analysis of the RT differences and a concerning issue that mix truthful and untruthful responses, we had participants play a message-sending task with self-interest temptation and mouse tracking (MT) technique. Potentially, the implication of temporal evidence with MT could be a supplemental way to gain mechanistic insight from delivering signals in communication. MT is an emerging technique to measure the real-time mouse movements during decision-making (J. Freeman, Dale, \& Farmer, 2011). It provides rich temporal information to reveal how decisions evolve, such as hesitating or changing mind halfway, and allow researchers to understand the time-course of a
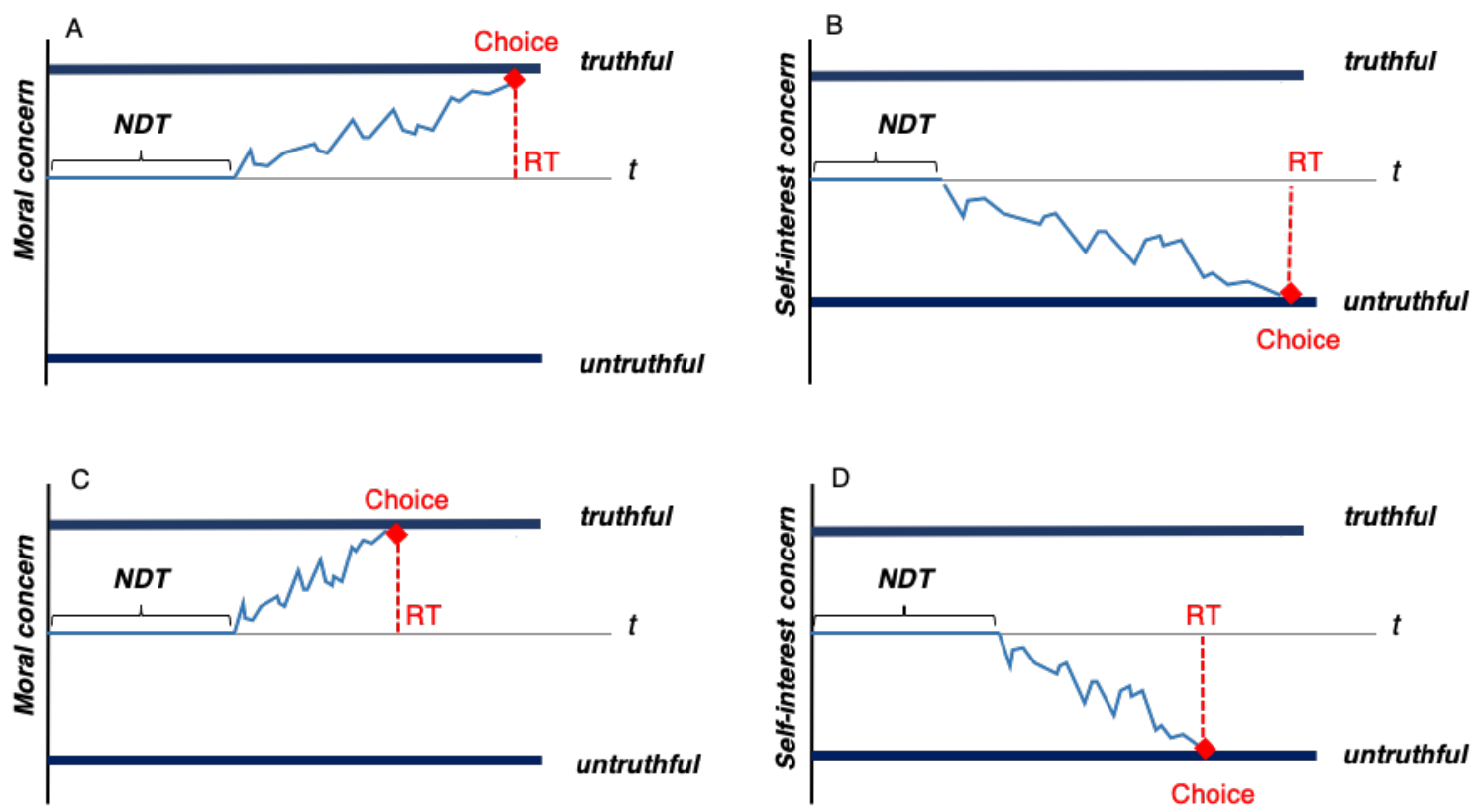

Figure 1 the hypothesis of possible DDM patterns in the dishonesty task decision process. What's more, a bunch of valuable information such as MAD (maximum deviation), AUC (area under the curve), spatial attraction/curvature, complexity, velocity, and acceleration provide more information in the dynamic of decision (J. B. Freeman \& Ambady, 
2010). So far, there are attempts to track hand or arm movement in truthful/untruthful responses (N. D. Duran, Dale, \& McNamara, 2010; Monaro, Gamberini, \& Sartori, 2017), but none of them considered a self-interest setting combining with computational modeling.

Taken together, the present study is to investigate the following novel hypothesis: 1) the fair default choice makes engaging in honest action is easier than in dishonest action in a selfinterest context, which depends on one's own moral tendency; 2) deliberative honesty/dishonesty is detectable with mouse tracking, which is also associated with one's weight on self-interest; 3) MT indices and computational modeling can contribute to the detection of self-interest weights in the dishonesty decision, which would explain personal selfinterest concern tendency. To test these hypotheses, we investigated honesty/dishonesty in signaling games with two experiments (one with moral default option in experiment1, and no default choice in experiment 2) and examined the cognitive mechanisms of multiple stages during honest/dishonest decisions. With the combination of utility model, DDM and MT indices, we predict effortful lying or deliberative honesty driven by self-interest. Besides, individual differences can be manifested on parameters of computational modeling and mouse trajectories.

\section{Experiment}

\section{Experiment 1}

In this experiment, we investigated whether people would like to make dishonest responses when there is a default (fair) option, and how mouse dynamics are reflecting their selfish tendency. We also predict the non-prolonged RT for spontaneous dishonest responses, but the drift rate in DDM can differentiate honesty vs. dishonesty. Our experiments have been pre-registrated in OSF( $\underline{\text { osf.io/wb5su}})$.

Participants. Eighty-one healthy undergraduate and graduate students (21 males, $M_{\mathrm{age}}=$ 23.1 years, $S D_{a s e}=3.22$ ) participated in this experiment. All participants were right-handed and had normal or corrected-to-normal vision. The participants were told that the points they earned during the game were positively correlated to their final payoff. At the end of the study, the participants were paid 20-30 yuan. All subjects signed informed consent before the formal experiment, and the experimental protocol was approved by the local ethical review committee.

Task and procedure. Before beginning the experiment, participants completed a practice to familiarize themselves with the mouse and decision task. In the experiment, we used a 
modified signaling game: a message task adopted from previous studies (Zhu et al., 2014). The game captures a core dilemma involved in honest behavior where the interests of the signaler conflict with those of the signal receiver. In the task, a signaler can choose to either truthfully disclose or misrepresent information about which one is the better choice for the receiver that eventually has direct monetary consequences for both sides (signaler and receiver).

Specifically, in the task, participants were instructed that they were randomly assigned as the sender or the receiver. The task consisted of a series of decisions in which the sender telling the receiver which choice benefits them more, and the sender was told that all information the receiver got was only their advice. However, in the trials with conflict of interest, the sender may decide to lie to gain more money for themselves, and the receiver may take the advice or not. In the experiment, all participants were assigned as the sender who can give suggestions to the fake receiver about which option contributes to more payoffs for the sender self or the counterpart receiver via self-other tradeoffs.

Before each of the testing session, the participants have first presented the same default option of "self: 50, other: 50", which means the sender and the receiver will get 50 points each if choose the default. With the mouse tracker software to record the mouse trajectories (J. B. Freeman \& Ambady, 2010), every trial will begin with a "start" in the middle bottom of the screen that the sender must press to begin the trial. Once the participant clicks "start", an allocation of money between themselves ("self") and the other player ("other") will be displayed. The participant can advise the receiver of the default "50:50", or instead advise them to take the "current" allocation. The "Default 50:50" allocation is presented to the sender only before each block. The receiver can follow the advice or reject the advice. To avoid the higherorder mentalizing here, there is no feedback of the receiver's final decision in the experiment. The sender is supposed to believe that receivers would never know whether a message they received is true or not. This highlights that the signal receiver is entirely reliant upon the sender for potential information about the options and prevents them from using payoff information to make inferences about signaler behavior (Gneezy, 2005). Participants completed 40 twochoice trials in total during the task. With the design of self-other interest conflicts, there are two conditions in the trials: the conflict trials (29 trials) vs. the non-conflict trials (11 trials). The trial order was randomized for all participants. The experiment consists of two blocks, with each block lasts around 10 minutes (see Figure 2). 


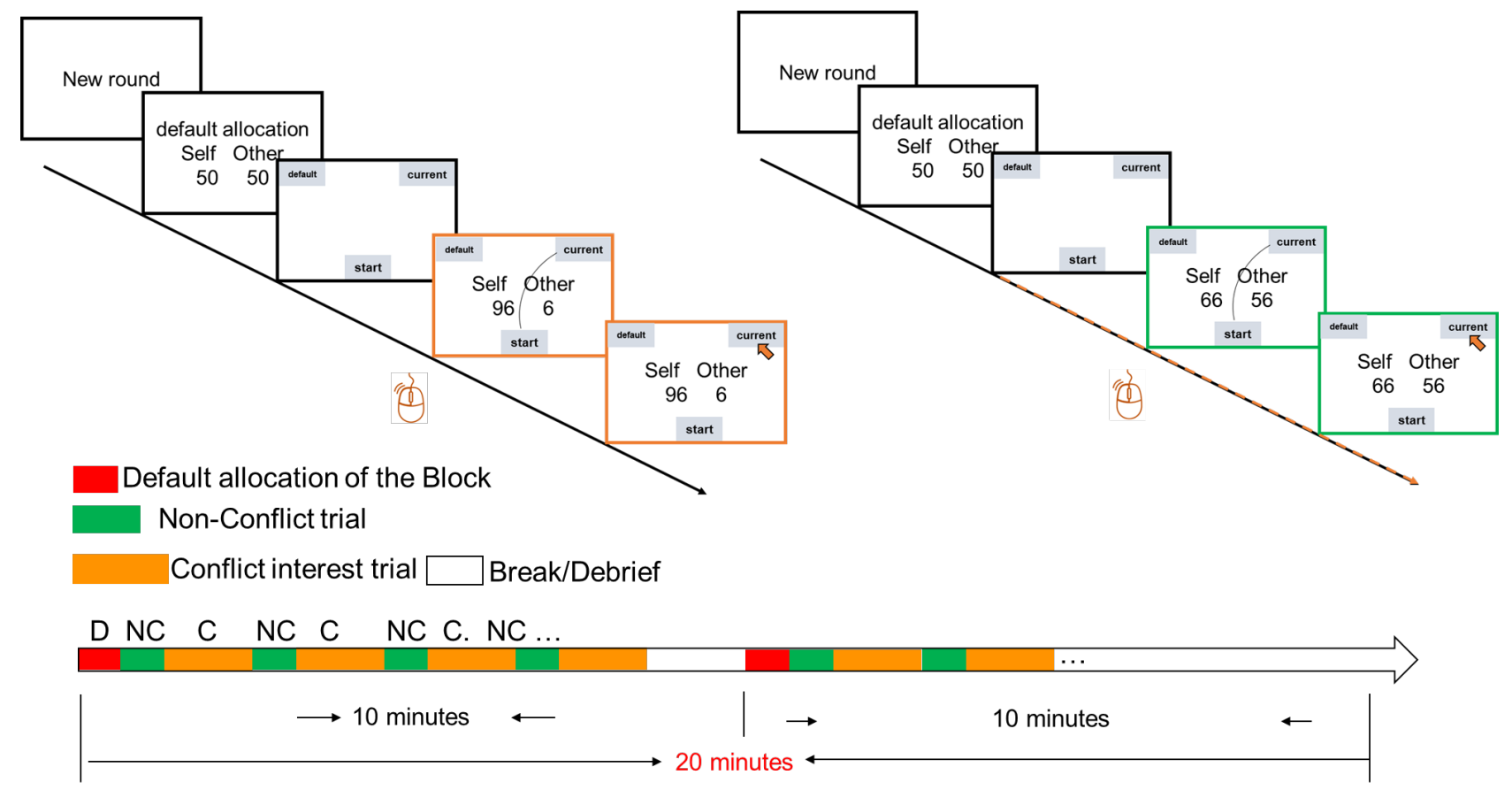

2 blocks

Figure 2. The procedure and block structure in experiment 1 $(\mathrm{C}=$ conflict trials; $\mathrm{NC}=$ noconflict trials $)$.

Most trials are conflict trials in the design and there is a default option in all trials

\section{Data analysis}

Defining dishonest/untruthful response

A reasonable way to define dishonest behavior in this task is where the value of the option that participants choose to send for the counterpart is lower than the other option. We first defined sending wrong information in conflict trials as dishonesty and calculated the dishonesty rate here as one of the dependent variables. In this case, we focus on conflict trials only because sending wrong information intentionally in conflict trials can be assumed as the self-interestmotivated dishonesty.

We are also concerned that individual choice in our study might also be a function of value difference between self and other or between two options for themselves, besides, as well as their prosociality. Under the self-interest temptation context, various value-based struggles affect participants' actions. Therefore, we have also used the computational modeling of the choices to define how much they concern about self and other's payoffs. 
The mouse-trajectory preprocessing is processed with $\mathrm{R}$ language and mousetrap package (version 3.5.2). Standard mouse-tracking preprocessing was used (J. B. Freeman \& Ambady, 2010). All response trajectories were rescaled into standard coordinate space (top left: $[-1,1.5]$; bottom right: [1,0]) and normalized into 100-time bins using linear interpolation to permit averaging of their full length across multiple trials. For comparison, all trajectories were remapped rightward. To obtain a trial-by-trial index of category co-activation, we calculated the MAD and AUC.

\section{Computational modeling of the choices}

To characterize the relative contributions of economic self-interest, distributional preference, and honesty consideration to message task decisions, we adapted an economic model that was previously applied to a similar task (for details, see Zhu et al., 2014). As shown in equation (1), the Ms and Mo are monetary payoffs for self and others respectively. The decision-maker's utility is modulated by honesty and monetary allocation to self and others. With a similar computational modeling approach, we got parameters capturing distributional preferences that solely depend upon the monetary allocation between self and others. In contrast, model parameter delta ( $\delta$, the larger, the more care about honesty) quantifies the bias effects of honesty concerns. The functional form follows the well-established Constant Elasticity of Substitution utility function. Specifically, the alpha $(\alpha)$ parameter quantifies the relative weight between monetary payoffs for self and others, the larger $\alpha$ indicates a larger weight on the own economic gain. To calibrate the model given the binary choice behavior, we adopted the standard logit assumption, aggregated observations conditional on and experimental conditions. We conducted the maximal likelihood estimation, specifically maximizing the loglikelihood function as shown in equation (2). The standard errors of estimated parameters were obtained through the bootstrap procedure with 2000 iterations.

$$
\begin{gathered}
U\left(M_{S}, M_{o}\right)=\left[(\alpha-I \cdot \delta) M_{s}^{\rho}+(1-\alpha+I \cdot \delta) M_{o}^{\rho}\right]^{\frac{1}{\rho}} \\
\sum_{i} \sum_{t} \log \left(P_{i, t}\left(y_{i t} ; \alpha_{M}, \alpha_{C}, \rho\right)\right)
\end{gathered}
$$

Drift diffusion modeling of the RTs

Unlike traditional analysis with the mean response times (RTs) and accuracy, the driftdiffusion modeling of the RT is processed with R language and hBayesDM package (version 3.1.1) (Ahn, Haines, Zhang, \& Ahn, 2016). We modeled the choice and RTs for four conditions 
from conflict (2: conflict vs. non-conflict) by responses (2: truthful vs. untruthful) design. With the individual Bayesian modeling of the task using DDM, we got the following parameters: alpha (boundary separation), beta (bias), drift rate for each condition, and tau (non-decision time). The parameters describe the specific psychological aspects (Voss et al., 2004), for example, the drift rate represents the average slope of the accumulation process towards the boundaries. The larger the drift rate, the stronger evidence accumulated for the corresponding response option. The starting point is a measure of response bias towards one of the two response boundaries. Non-decision time captures all non-decisional processes such as stimulus encoding and response processes.

In the simple DDM of our study, the drift rate of each condition can be considered as a measure of task difficulty (in the comparison of task conditions) or cognitive speed (in the comparison of individuals). We aimed to test the cognitive speed difference between conflicttruthful and conflict-untruthful responses and their relationship with moral tendency.

Statistical analysis

The RTs were analyzed in a mixed-effects model, including the fixed interaction effect condition (conflict/non-conflict) and response (truthful/untruthful), as well as the intercepts for the subject of the random effects.

To model the RT and the reward difference between these two options, we have also implemented the mixed-effects model with the value difference of self-payoff, and the value difference of other-payoff by random subject slopes for the effect of RT. The linear mixedeffects analysis was carried out using the lmer () function from the lme4 package (Bates, Mächler, Bolker, \& Walker, 2014) as well as the anova () function from the stats package (Team, Team, Suggests, \& Matrix, 2013).

Degrees of freedom were approximated using Satterthwaite's method. Post-hoc tests were carried out using a Tukey-test. Bonferroni adjustment of p-values was done via the glht() function from the multcomp package (Team et al., 2013) in R version 3.5.2 (R Core Team 2014). To examine whether the decision-making time associates with one's selfish tendency, the correlation analysis was also implemented between DDM parameters, trajectory indices and utility model parameters in experiment 2.

\section{Results}

Choice percentage. 
For the conflict trials that we focus on most, the mean dishonesty rate (as defined in section 3.1 ) is $38.2 \%$, with $61.8 \%$ truthful responses.

A mixed model of $R T$.

For the mixed model analysis of the RT with conflict and response effects, we found a significant effect of conflict on the RTs in experiment 1 . The conflict effect indicated that RTs in the conflict trials were shorter than non-conflict trials, $F_{3151}=-2.036, p=0.042$.

Truthful vs. untruthful responses under conflict trials

As our specific interest is the comparison between truthful and untruthful responses for conflict trials, we directly performed the t-test for the RTs and mouse trajectory indices (MAD and AUC) between the two conditions. The results did not show significant difference between the two conditions on both the RTs $(t=-0.31699, d f=159.99, p=0.7517)$ and mouse trajectory indices (e.g., MAD: $t=0.16405, d f=158.92, p=0.8699$; AUC: $t=1.0507, d f=$ $141.97, p=0.2952$ ), $P \mathrm{~s}>.254$ (see plots in Figure $S 1$ ).

\section{Correlations among self-interest concern, $R T$, and trajectory indices}

As for the correlations among the modeling parameter and the RTs, we found a significant positive correlation between the self-interest concern parameter (alpha) and the RTs under the conflict-truthful response ( $r=0.24, p=0.033)$, indicating more concern about self-interest, the longer time is needed to make the truthful response in conflict trials (See Figure 3A). Regarding the correlations among the utility modeling parameter and the trajectory indices, we observed a significant negative correlation between the self-interest concern parameter (alpha) and the MAD $(r=-0.24, p=0.033)$ and AUC $(r=-0.25, p=0.022)$ for untruthful responses in conflict trials (See Figure 3B \& Figure 3C). 

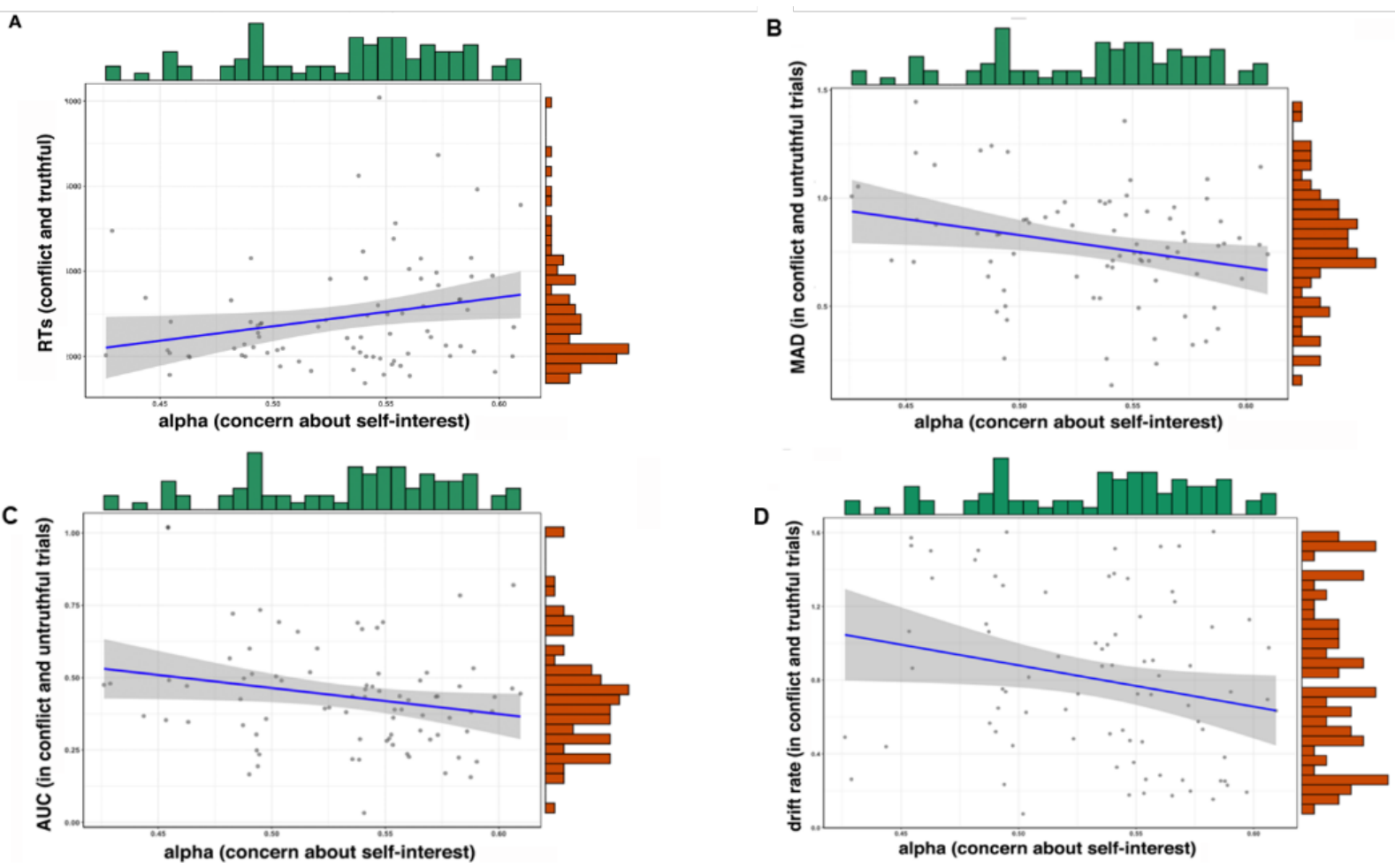

Figure 3 Correlations between the self-interest concern parameter (alpha) and behavioral dependent variables

A) The positive correlation between the self-interest concern parameter (alpha) and RTs of a truthful decision in conflict trials; B) a negative correlation between the self-interest concern and trajectory index MAD of an untruthful decision in conflict trials; C) a negative correlation between self-interest concern and trajectory index AUC of an untruthful decision in conflict trials; D) a negative correlation between selfinterest concern and drift rate of a truthful decision in conflict trials.

\section{DDM model index in the conflict trials}

Unlike the RT results, we observed that the drift rate of truthful responses in conflict trials was significantly higher than the conflict-untruthful responses, $t=4.4739, d f=151.07$, $p<0.0001$ (see Figure 4). There was also a significant negative correlation between alpha (concern about self-interest) and drift rate in conflict-truthful responses (see Figure 3D), $r=-$ $0.23, t=-2.0853, d f=79, p$-value $=0.04$. 

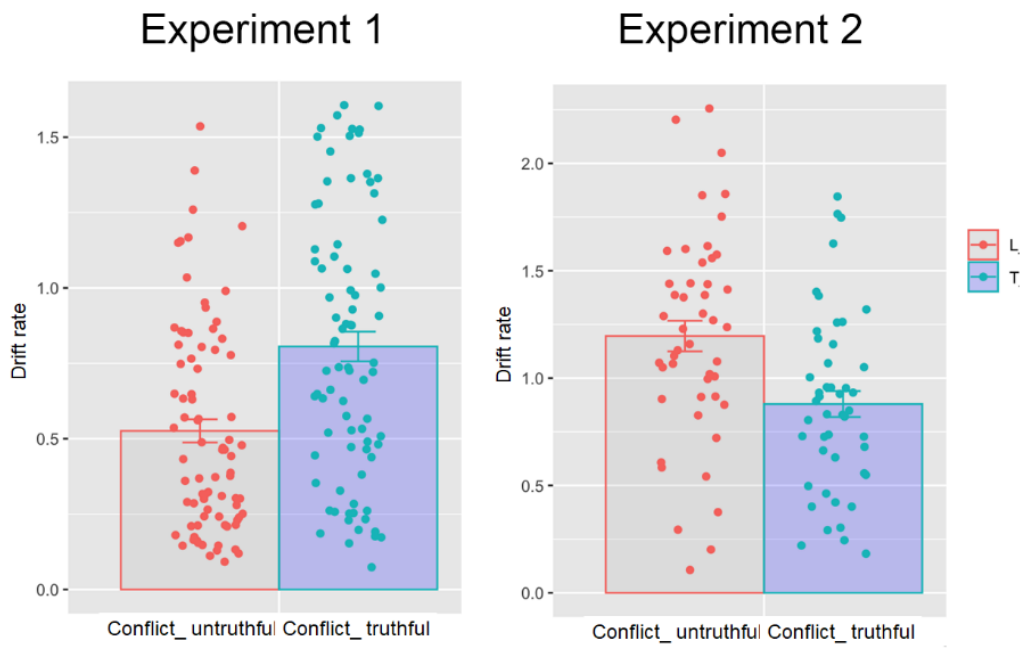

Figure 4. The drift rate $(v)$ for conflict_unthuthful condition and conflict_thruthful

condition in experiment 1 and experiment $2(\mathrm{~L}=$ untruthful, $\mathrm{T}=$ truthful $)$. It indicates higher drift rate conflict_truthful condition in Experiment 1, while low drift rate conflict_truthful condition than conflict_untruthful in Experiment 2

\section{Experiment 2}

In this experiment, we investigated how people make decisions using a two-alternative forced-choice design rather than default(fair) option task and replicated that mouse dynamics indices associated with their expressed selfish tendency in experiment 1 . We tested both the RTs for spontaneous dishonest responses and the drift rate $(v)$ in the DDM differentiation effect between honest and dishonest decisions. We have also done pre-registration in open science framework (osf.io/wb5su).

Participants. Forty-seven healthy undergraduate and graduate students (19 males, $M_{\mathrm{age}}=$ 22.78 years, $S D=2.89$ ) participated in this experiment. All participants were right-handed and had normal or corrected-to-normal vision. The participants were told that the points they earned during the game were related to their final payoff. At the end of the study, the participants were paid 20-40 yuan. All subjects signed informed consent before the formal experiment. The experimental protocol was approved by the local and Caltech ethical review committee (IRB:18-0790).

Procedure. The mouse tracking protocol was adapted based on experiment 1, except that we changed the payoffs into two options of A (e.g., self: 13, other: 3) and B (e.g., self: 7, other: 5) (see Figure 6), rather than using default and current options. The participant was asked to send the option that would make more money for the other counterpart, and the 
payoff space of the trials was plotted in Figure 6A. There were 88 conflict trials out of 120 trials in total. The trial order was randomized for all participants. The experiment consists of two blocks, with each block lasts around 30 minutes (see Figure 5).
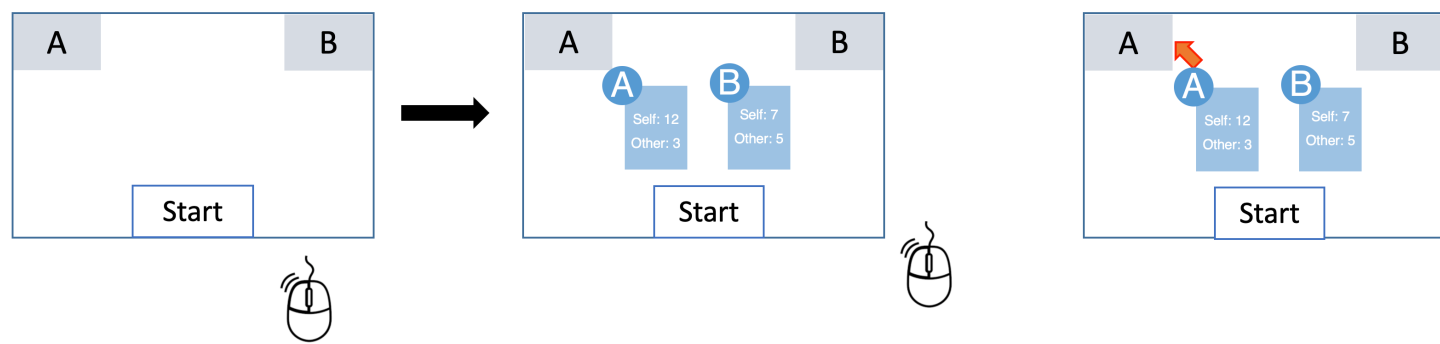

Non-Conflict trial

Conflict interest tria

Break/Questionnaire time

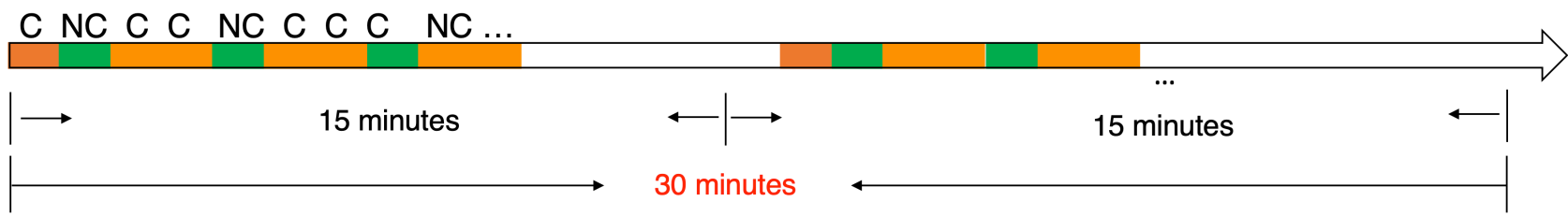

Figure 5. Task and procedure for experiment 2

$(\mathrm{C}=$ conflict trials $; \mathrm{NC}=$ noconflict trials $)$.

Most trials are conflict trials in the design and there are two options (A and B) with no default option

in all trials

\section{Data analysis}

The data (RTs, MTs, DDM and utility model) of experiment 2 was analyzed in the same way as the Experiment 1.

\section{Results}

Choice percentage.

For the conflict trials that we focus on most, the mean dishonesty rate (defined in section 3.1 ) is $58.3 \%$, with $41.7 \%$ truthful responses.

\section{Mixed model of RTs}

For the mixed model analysis of the RTs with conflict and response effects, we found a significant interaction effect of conflict and response in experiment $2, F_{5247}=-6.124, P<$ 0.001 . The interaction effect $\left(F_{5713}=-8.644, P<0.001\right)$ indicated that untruthful responses 
under conflict trials exhibited shorter RTs than non-conflict trials, while RTs of truthful response under conflict trials were longer than non-conflict trials (see Figure 6D).
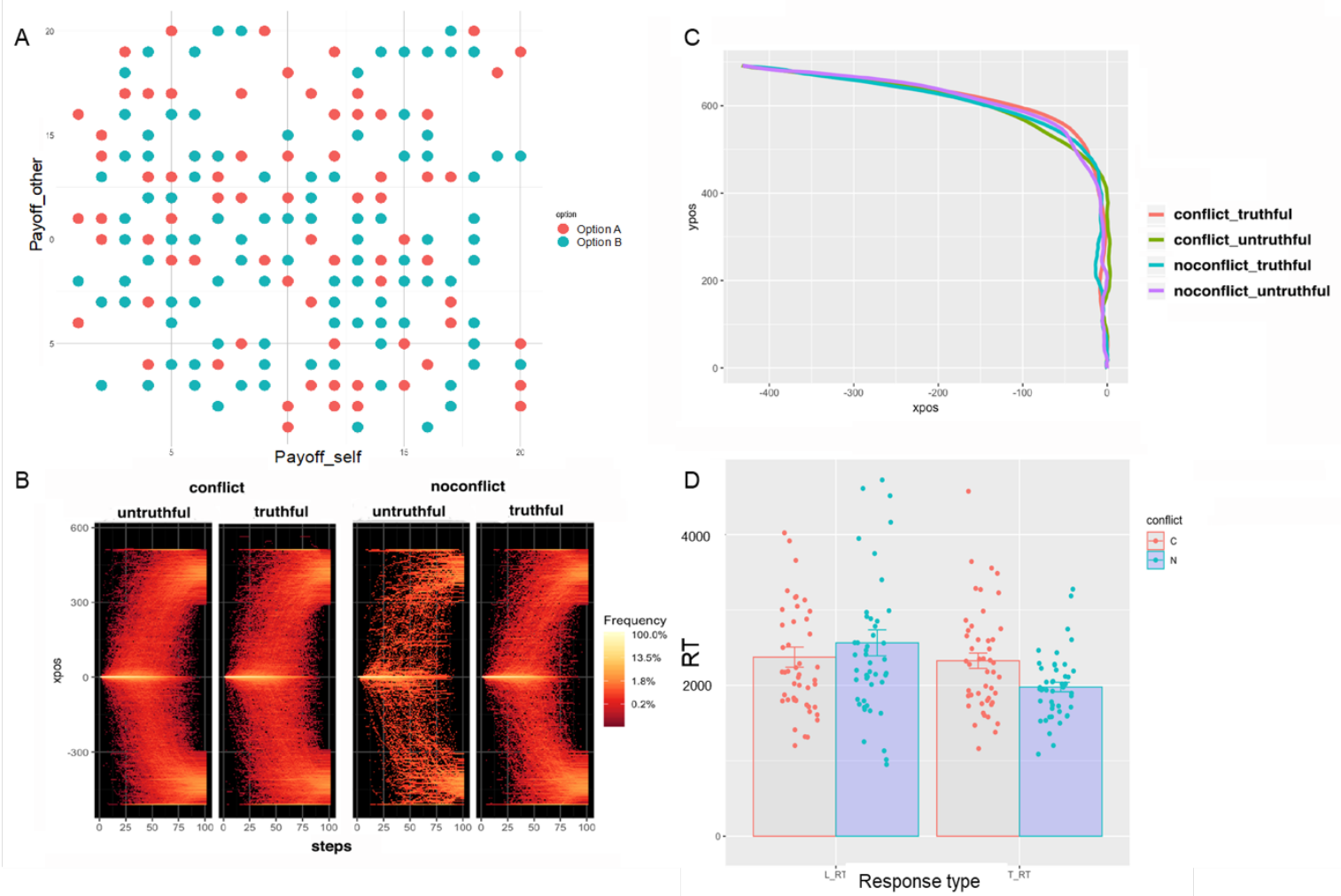

Figure 6. The set up and initial results in Experiment 2. A) The payoff space in Experiment 2; B) the mouse trajectories maps in Experiment 2; C) the density of mouse positions across 100 time bins; D) RTs of truthful response under conflict trials were faster than non-conflict trials $(\mathrm{L}=$ untruthful, $\mathrm{T}=$ truthful, $\mathrm{C}$ $=$ conflict, $\mathrm{N}=$ noconflict $)$.

Truthful vs. untruthful responses under conflict trials

As our specific interest is the comparison between truthful and untruthful responses for conflict trials, we directly performed the t-test for the RTs and mouse trajectory indices (MAD and AUC) between these two conditions. The results did not show significant difference between these two conditions on the RTs $(t=0.28, d f=85.94, p=0.78)$, while we found a significant larger trajectory AD (averaged deviation) for the conflict-truthful trials than the conflict-untruthful trials, $t=-2.5392, d f=86.929, p=0.012$. Similarly, we also found a larger trajectory AUC for the conflict-truthful trials than the conflict-untruthful trials, $t=-1.9999, d f$ $=87.243, p=0.048$.

Correlations among self-interest concern, RT, and trajectory indices 
As for the correlations among the modeling parameters and the RTs, we found a significant negative correlation between the self-interest concern parameter (alpha) and the RT under the conflict-untruthful response $(r=-0.54, p<0.001)$, indicating more concern about self-interests, the longer time needed to make the truthful response in conflict trials There was no significant correlation between the self-interest concern parameter (alpha) and the RT under the conflict-truthful response $(r=-0.15, p=0.322)$.

Regarding the correlations between the utility modeling parameters and the trajectory indices, we observed significant negative correlations between the self-interest concern parameter (alpha) and the MAD $(r=-0.30, p=0.042)$, and correlation between self-interest concern parameter (alpha) and AUC $(r=-0.31, p=0.037)$ for untruthful responses in conflict trials.

\section{Drift rate in DDM models in the conflict trials}

Unlike the RT results, we observed that the drift rate of untruthful responses in conflict trials was significantly higher than the conflict-truthful responses in experiment 2 (see Figure 4), $t=4.4739, d f=151.07, p<0.0001$. There was also a significant negative correlation between alpha (concern about self-interest) and drift rate in conflict-truthful responses (see Figure 3D), $r=-0.23, t=-2.09, d f=79, p<0.05$.

Further, we have also observed the significant negative correlation between concern about self-interest(alpha) and the drift rate in conflict but truthful response, $r=-0.57, t=-$ 4.6818, $d f=45, p<0.0001$, while a positive correlation between concern about selfinterest(alpha) and the drift rate in conflict but untruthful response, $r=0.71, t=6.77, d f=45$, $p<0.0001$. Furthermore, there was significant negative correlation between MAD and the drift rate in conflict but untruthful response, $r=-0.34, t=-2.4421, d f=45, p<0.05$.

\section{Discussion}

Dishonesty is a computationally demanding process, which requires the integration of information in the context of goals, expectations, preferences, and other factors. The present study follows the previous Will and Grace hypothesis to investigate the behavioral responses (RT and mouse trajectories) and individual differences of dishonesty in settings with and without default settings. For the Grace hypothesis, people are per internalized social norms (e.g., honesty, fairness) which shapes the moral default. However, when self-interest is 
involved, honest individuals display moral default with more hesitation in dishonesty while self-interest-driven individuals are faster to make an untruthful response (i.e., Will hypothesis). We observed how motor action indicates tradeoffs under conflicts between self and others and its correlation with moral tendency. Further, rewards play a crucial role when there is a chance to be dishonest: the higher the reward, the more temptation to be dishonest. Consistent with this view, our utility modeling results and MT indices show that selfish people will engage in such violations more often and give us more insights into dynamics in which self-interest trades off with honesty in making decisions. Our findings partially confirm our predictions that moral default could be modulated by self-interest temptation, with some interesting evidence via mouse tracking (e.g., more hesitation in trajectory index for truthful response in conflict condition).

Indeed, we observe lower dishonesty rate and shorter RTs in experiment 1 with the default setting than experiment 2 , which seems to support the moral default hypothesis that dishonesty requires cognitive control to suppress the moral response. However, there is a debate about whether dishonesty requires a longer time because, while dishonesty could be the automatic response with the motivation to pursue self-interest (J. D. Greene \& J. M. Paxton, 2009). We do find evidence that people show dishonesty when there is conflict of interest in both experiment 1 and experiment 2. Dishonesty can be more spontaneous when self-interest is confronted with others' benefits when there was conflict interest between self and others, which is consistent with the previous research. Intriguingly, untruthful responses under conflict trials displayed shorter RTs than non-conflict trials. Contrary to our common sense, conflict situations produced less reactional hesitation. Our results show participants in conflict manipulation setting took shorter time than non-conflict settings to choose in experiment 1 , but not in experiment 2. It is possible that the difference between each option is small in which the decision difficulty increases, and participants take more time to think (Westbrook, Lamichhane, \& Braver, 2019). This study did not find a stable difference of RTs between truthful vs. untruthful responses in a conflict vs. noconflict situation. Because there are not many non-conflict trials in experimental 1 and 2, it provides inadequate data for us to analyze the RT difference between the conditions. Besides, the self-involved dishonest decision is not the same as the simple lying process, and the RT measures may not capture the difference between truthful and untruthful responses.

Although the RT difference in two contexts is not significant in experiment 2, the mouse trajectory index - AUC indicate a similar pattern that a larger AUC for truthful responses than 
untruthful responses in the conflict condition. The visible hand movements in current findings can enrich our understanding of the cognitive process under dishonesty (Monaro et al., 2017) in self-serving context. These findings show that in a conflict situation, truthful responses require greater effort and cognitive control as evidenced by longer RTs than untruthful responses. While in a non-conflict setting, truthful responses appeared to be easier because a deception act needs to resolve competition between truth-telling and false-message-sending (Nicholas D. Duran, 2010).

With a self-serving context to obtain monetary rewards, Speer et al. (2020) found that participants with a higher rate of cheating exhibited increased brain activation in cognitive control brain regions such as the anterior cingulate cortex (ACC), and the inferior frontal gyrus (IFG), when making decisions to be honest. Overall, these findings emphasize that higher rewards and greater sensitivity to own interest increase the likelihood of dishonesty. Computationally, we found a positive correlation between the parameter $\alpha$ fitted by utility modal (concern about self-interests) and RT to make the truthful response in conflict trials. Longer RTs of truthful action are associated with greater reward concern of oneself and the suppression of self-serving motivation. From another view to understand the mental process when weighing two options, the RTs pattern was also presented in our mouse tracking results. The parameter $\alpha$ of the utility model was negatively correlated with the AUC and MAD in conflict trials when making dishonest responses. A large $\alpha$ indicates a larger weight on the economic gain and MAD/AUC indicates an increased complexity in one responding relative to the other. In this case, a larger MAD/AUC reflects the dynamic bias at the initial stage of untruthful decision-making. This bias results from a counter-intuitive bias to be honest when making a dishonest choice, while such bias did not show in the truthful response in a conflict context. The above results vividly reflect a mental process about self-interest involved decision when making a (un)truthful decision. First, conscience is alerting to repel our selfish mind when making dishonest responses and could be detectable by hand movement with a more complex motor pattern. Secondly, it also requires effort when self-focused individuals give up their benefits to make a truthful response.

In addition to examining the dynamic motor processes, we examined the dynamic processes of evidence accumulation from the perspective of perceptual processing. Typically, models of evidence accumulation can be used to describe decision-making dynamics. When decision-making is a continuous process that fluctuates between two possible options, DDM has the advantage of statistically separating distinct aspects of a decision process. The drift rate 
in DDM represents the speed of evidence accumulation used for the decision. Generally, the drift rate is lower for difficult condition than easier conditions (modulated by motion coherence) (Bottemanne \& Dreher, 2019). Our study observed different pattern of drift rate for truthful and untruthful responses in experiment 1 and experiment 2, which indicate evidence accumulation of truthful response is faster than untruthful responses for conflict trials in experiment 1, with default option. This pattern is reversed in experiment 2 , there are two options to choose, which may facilitate the self-interest motivation. However, both experiments show a significant negative correlation between alpha (concern about self-interest) and drift rate in conflict-truthful responses (see Figure 3D). Like food or healthy choices, people may regulate dietary behavior by changing the weights to specific attributes (tastiness vs. healthiness)(N. Sullivan, Hutcherson, Harris, \& Rangel, 2015). It seems that the evidence integration is faster in conflict trials with default option, which may indicate the self-interest drives fast integration in the value-based moral decision. In this vein, payoffs for self and others integrate into a single source of evidence quickly and the negative correlation between selfinterest concern and drift rate value of conflict-truthful response further endorse this interpretation. As the recent work indicated that cheaters applying more cognitive control led to less dishonesty while, the more cognitive control for more honest participants, the more likely it is they will cheat (S. P. Speer et al., 2021). Here our RTs and DDM results further confirm of individual difference on moral default, and individual's weight of self-interest are associated with effort and speed in one's (dis)honesty decisions. That is, individuals who are more selfish focus on self-payoffs only and integrate the information faster, showing the reward processing influenced by individual self-focused tendency may predict individual differences in dishonesty (N. Abe \& Greene, 2014).

Several limitations could be addressed in future studies. In our results, no stable RT difference between conflict and no-conflict is observed and we find that truthful response is longer in a conflict context when a fair default option is presented. This raises a question of whether people make untruthful decisions derived from a goal-directed or it is just a casual mistake because the truthful and untruthful definition is an after-mind categorization. For instance, it is reported that an observed prosocial decision may just a beautiful mistake during a decision instead of deliberative kindness (Hutcherson, Bushong, \& Rangel, 2015). Systematic RT consistency in conflict context may not reflect the same underlying preferences (i.e., weights on being true and untrue), but simply alternation in the same amount of noise in the decision process. So far, we have only been able to find differences between dishonest and 
honest behavior at the level of behavioral indicators. However, a major concern is the extent to which a spatial trajectory analysis reflects the attention or cognitive control of a mental process, which is beyond the focus of this work. As mouse tracking offers valuable temporal motion information, future research is encouraged to combine mouse-tracking technology with other high time resolution techniques (e.g., EEG) or eye-tracking techniques to plays a crucial role in measuring individual dynamic cognitive processes in real-time.

Overall, by employing emerging computational models and mouse-tracking, we shed light on dishonesty decisions associated with self-interest concerns. Generally, mental processes about the strength of competing between being honesty and benefit-maximization could be vividly demonstrated in our dynamic motor measurement. Since the relation between perceptual input and motor response is dynamic and continuous, the information processed is transmitted to the motor cortex continuously in the form of mouse movement, and in turn, guides our movement in real-time (Freeman et al., 2011). Under this assumption and our observations of RTs, mouse tracking and model parameters, we are confident that our results based on cognition-action interplay reveals the individual difference in default moral responses when monetary temptation is presented. With the introduction of computational models, we can decompose a potential information sampling process during (dis)honesty decisions and tell apart how do the decisions happen under the temptation of self-interest context. Besides, less RTs or struggle in conflict conditions was replicated across two experiments. Meanwhile, the present study makes up for the lack of the previous discussion about factors of moral default. We show dishonesty tendency were correlated with individual differences in the self-interest weight. This framework enlightens our understanding of dishonest behavior and offer a novel observation of self-interest motives to moral decisions with motor responses.

\section{Acknowledgments}

The authors thank Hao Yu, Shanshan He and Ning Zang for assistance with data collection.

This work was supported by National Natural Science Foundation of China (U1736125 to H.W.), National Institute of Mental Health grant2P50MH094258, a Chen Institute award (P2026052), and Templeton Foundation grant TWCF0366 (all to D.M.)

\section{Data and code availability}

The data and code that support the findings of this study are available at https://github.com/andlab-um/MTbeha.

\section{Declaration of Conflicting Interests}


The authors declared that they had no conflicts of interest with respect to their authorship or the publication of this article.

\section{Funding}

This work was supported by National Natural Science Foundation of China (U1736125) and

SRG of the University of Macau.

\section{References}

Abe, N. (2011, 2011/10/01). How the Brain Shapes Deception: An Integrated Review of the Literature. The Neuroscientist, 17(5), 560-574. https://doi.org/10.1177/1073858410393359

Abe, N., \& Greene, J. D. (2014, Aug 6). Response to Anticipated Reward in the Nucleus Accumbens Predicts Behavior in an Independent Test of Honesty. Journal of Neuroscience, 34(32), 10564-10572. https://doi.org/10.1523/Jneurosci.0217-14.2014

Ahn, W.-Y., Haines, N., Zhang, L., \& Ahn, M. W.-Y. (2016). Package 'hBayesDM'.

Ariely, D., \& Jones, S. (2012). The (honest) truth about dishonesty. Harper Collins Publishers New York.

Atherton, G., Lummis, B., Day, S. X., \& Cross, L. (2019, Jul). What am I thinking? Perspective-taking from the perspective of adolescents with autism. Autism, 23(5), 11861200. https://doi.org/10.1177/1362361318793409

Baron-Cohen, S., Wheelwright, S., Skinner, R., Martin, J., \& Clubley, E. (2001). The autismspectrum quotient (AQ): Evidence from asperger syndrome/high-functioning autism, malesand females, scientists and mathematicians. Journal of autism and developmental disorders, 31(1), 5-17.

Bates, D., Mächler, M., Bolker, B., \& Walker, S. (2014). Fitting linear mixed-effects models using lme4. arXiv preprint arXiv:1406.5823.

Bereby-Meyer, Y., \& Shalvi, S. (2015). Deliberate honesty. Current Opinion in Psychology, 6, 195-198.

Bottemanne, L., \& Dreher, J. C. (2019). Vicarious Rewards Modulate the Drift Rate of Evidence Accumulation From the Drift Diffusion Model. Frontiers in Behavioral Neuroscience, 13. doi:ARTN 14210.3389/fnbeh.2019.00142

Busemeyer, J. R., \& Johnson, J. G. (2004). Computational models of decision making. Blackwell handbook of judgment and decision making, 133-154.

Capraro, V. (2017, Sep). Does the truth come naturally? Time pressure increases honesty in one-shot deception games. Economics Letters, 158, 54-57.

https://doi.org/10.1016/j.econlet.2017.06.015

Christ, S. E., Van Essen, D. C., Watson, J. M., Brubaker, L. E., \& McDermott, K. B. (2009). The Contributions of Prefrontal Cortex and Executive Control to Deception: Evidence from 
Activation Likelihood Estimate Meta-analyses. Cerebral Cortex, 19(7), 1557-1566. https://doi.org/10.1093/cercor/bhn189

Duran, N. D. (2010). The action dynamics of overcoming the truth. Psychonomic Bulletin \& Review, 17(4), 486-491.

Duran, N. D., Dale, R., \& McNamara, D. S. (2010, Aug). The action dynamics of overcoming the truth. Psychon Bull Rev, 17(4), 486-491.

https://doi.org/10.3758/PBR.17.4.486

Freeman, J., Dale, R., \& Farmer, T. (2011). Hand in motion reveals mind in motion. Frontiers in Psychology, 2, 59.

Freeman, J. B., \& Ambady, N. (2010). MouseTracker: Software for studying real-time mental processing using a computer mouse-tracking method. Behavior research methods, 42(1), $226-241$.

Freeman, J. B., Dale, R., \& Farmer, T. A. (2011). Hand in motion reveals mind in motion. Frontiers in Psychology, 2. https://doi.org/ARTN 59

10.3389/fpsyg.2011.00059

Gneezy, U. (2005). Deception: The role of consequences. American Economic Review, 95(1), 384-394.

Greene, J. D., \& Paxton, J. M. (2009). Patterns of neural activity associated with honest and dishonest moral decisions. Proceedings of the National Academy of Sciences, 106(30), 12506. https://doi.org/10.1073/pnas.0900152106

Happé, F. (2015). Autism as a neurodevelopmental disorder of mind-reading. Journal of the British Academy, 3. https://doi.org/10.5871/jba/003.197

Hutcherson, C. A., Bushong, B., \& Rangel, A. (2015). A neurocomputational model of altruistic choice and its implications. Neuron, 87(2), 451-462.

Levine, E. E., \& Schweitzer, M. E. (2014, Jul). Are liars ethical? On the tension between benevolence and honesty. Journal of Experimental Social Psychology, 53, 107-117. https://doi.org/10.1016/j.jesp.2014.03.005

Li, A. S., Kelley, E. A., Evans, A. D., \& Lee, K. (2011, Feb). Exploring the Ability to Deceive in Children with Autism Spectrum Disorders. Journal of Autism and Developmental Disorders, 41(2), 185-195. https://doi.org/10.1007/s10803-010-1045-4

Lu, H. Y., Li, P. L., Fang, J., \& Yi, L. (2019, Nov). The Perceived Social Context Modulates Rule Learning in Autism. Journal of Autism and Developmental Disorders, 49(11), 46984706. https://doi.org/10.1007/s10803-019-04174-y

Ma, W. N., Sai, L. Y., Tay, C., Du, Y. H., Jiang, J., \& Ding, X. P. (2019, Aug). Children with Autism Spectrum Disorder's Lying is Correlated with Their Working Memory But Not Theory of Mind. Journal of Autism and Developmental Disorders, 49(8), 3364-3375. https://doi.org/10.1007/s10803-019-04018-9 
Mahdi, S., Viljoen, M., Yee, T., Selb, M., Singhal, N., Almodayfer, O., Granlund, M., de Vries, P. J., Zwaigenbaum, L., \& Bolte, S. (2018, Mar). An international qualitative study of functioning in autism spectrum disorder using the World Health Organization international classification of functioning, disability and health framework. Autism Research, 11(3), 463475. https://doi.org/10.1002/aur.1905

Monaro, M., Gamberini, L., \& Sartori, G. (2017, May 18). The detection of faked identity using unexpected questions and mouse dynamics. PLoS One, 12(5). https://doi.org/ARTN e0177851

Overskeid, G. (2016, Aug 31). Power and Autistic Traits. Frontiers in Psychology, 7. https://doi.org/ARTN 1290 10.3389/fpsyg.2016.01290

Passanisi, A., \& Di Nuovo, S. (2015, Jan-Jun). Social and pragmatic impairments in individuals with Autism Spectrum Disorder. A lack of Theory of Mind? Life Span and Disability, 18(1), 75-99. < Go to ISI >://WOS:000420431400003

Pirrone, A., Dickinson, A., Gomez, R., Stafford, T., \& Milne, E. (2017, Feb). Understanding Perceptual Judgment in Autism Spectrum Disorder Using the Drift Diffusion Model. Neuropsychology, 31(2), 173-180. https://doi.org/10.1037/neu0000320

Pirrone, A., Johnson, I., Stafford, T., \& Milne, E. (2020, Mar 3). A diffusion model decomposition of orientation discrimination in children with Autism Spectrum Disorder (ASD). European Journal of Developmental Psychology, 17(2), 213-230. https://doi.org/10.1080/17405629.2018.1561364

Ratcliff, R., \& McKoon, G. (2008, Apr). The diffusion decision model: Theory and data for two-choice decision tasks. Neural Computation, 20(4), 873-922. https://doi.org/DOI 10.1162/neco.2008.12-06-420

Shalvi, S., Eldar, O., \& Bereby-Meyer, Y. (2012, Oct). Honesty Requires Time (and Lack of Justifications). Psychological Science, 23(10), 1264-1270.

https://doi.org/10.1177/0956797612443835

Speer, S. P., Smidts, A., \& Boksem, M. A. (2021). Cognitive control promotes either honesty or dishonesty, depending on one's moral default. Journal of Neuroscience.

Speer, S. P. H., Smidts, A., \& Boksem, M. A. S. (2020). Cognitive control increases honesty in cheaters but cheating in those who are honest. Proceedings of the National Academy of Sciences of the United States of America, 117(32), 19080-19091. doi:10.1073/pnas.2003480117

Suchotzki, K., Verschuere, B., Van Bockstaele, B., Ben-Shakhar, G., \& Crombez, G. (2017). Lying takes time: A meta-analysis on reaction time measures of deception. Psychological Bulletin, 143(4), 428-453. https://doi.org/10.1037/bul0000087 
Sullivan, N., Hutcherson, C., Harris, A., \& Rangel, A. (2015). Dietary Self-Control Is Related to the Speed With Which Attributes of Healthfulness and Tastiness Are Processed.

Psychological Science, 26(2), 122-134. doi:10.1177/0956797614559543

Sullivan, N. J., \& Huettel, S. A. (2021). Healthful choices depend on the latency and rate of information accumulation. Nat Hum Behav. doi:10.1038/s41562-021-01154-0

Talwar, V., Zwaigenbaum, L., Goulden, K. J., Manji, S., Loomes, C., \& Rasmussen, C. (2012, Jun). Lie-Telling Behavior in Children With Autism and Its Relation to False-Belief Understanding. Focus on Autism and Other Developmental Disabilities, 27(2), 122-129. https://doi.org/10.1177/1088357612441828

Team, R. C., Team, M. R. C., Suggests, M., \& Matrix, S. (2013). Package “Stats.”. RA Lang. Environment Stat. Comput. Vienna, Austria: R Foundation for Statistical Computing.

van Tiel, B., Deliens, G., Geelhand, P., Oosterwijk, A. M., \& Kissine, M. (2021, Jan). Strategic Deception in Adults with Autism Spectrum Disorder. Journal of Autism and Developmental Disorders, 51(1), 255-266. https://doi.org/10.1007/s10803-020-04525-0

Voss, A., Rothermund, K., \& Voss, J. (2004). Interpreting the parameters of the diffusion model: An empirical validation. Memory \& cognition, 32(7), 1206-1220.

Vrij, A., Fisher, R., Mann, S., \& Leal, S. (2006). Detecting deception by manipulating cognitive load. Trends in Cognitive Sciences, 10(4), 141-142.

https://doi.org/10.1016/j.tics.2006.02.003

Vrij, A., Granhag, P. A., Mann, S., \& Leal, S. (2011). Outsmarting the Liars: Toward a Cognitive Lie Detection Approach. Current Directions in Psychological Science, 20(1), 2832. https://doi.org/10.1177/0963721410391245

Walczyk, J. J., Harris, L. L., Duck, T. K., \& Mulay, D. (2014). A social-cognitive framework for understanding serious lies: Activation-decision-construction-action theory. New Ideas in Psychology, 34, 22-36.

Westbrook, A., Lamichhane, B., \& Braver, T. (2019). The Subjective Value of Cognitive Effort is Encoded by a Domain-General Valuation Network. The Journal of Neuroscience, 39(20), 3934. doi:10.1523/JNEUROSCI.3071-18.2019

Williams, D. M., Nicholson, T., Grainger, C., Lind, S. E., \& Carruthers, P. (2018, Aug). Can you spot a liar? Deception, mindreading, and the case of autism spectrum disorder. Autism Research, 11(8), 1129-1137. https://doi.org/10.1002/aur.1962

Xu, Z. X., \& Ma, H. K. (2015, 2015/03/01). Does Honesty Result from Moral Will or Moral Grace? Why Moral Identity Matters. Journal of Business Ethics, 127(2), 371-384. https://doi.org/10.1007/s10551-014-2050-x

Zhang, Y. X., Song, W. X., Tan, Z. L., Zhu, H. L., Wang, Y. Y., Lam, C. M., Weng, Y. F., Hoi, S. P., Lu, H. Y., Chan, B. S. M., Chen, J. J., \& Yi, L. (2019, Sep). Could social robots facilitate children with autism spectrum disorders in learning distrust and deception?

Computers in Human Behavior, 98, 140-149. https://doi.org/10.1016/j.chb.2019.04.008 
Zhu, L., Jenkins, A. C., Set, E., Scabini, D., Knight, R. T., Chiu, P. H., King-Casas, B., \& Hsu, M. (2014). Damage to dorsolateral prefrontal cortex affects tradeoffs between honesty and self-interest. Nature neuroscience, 17(10), 1319-1321. 
Figure $\mathrm{S} 1$
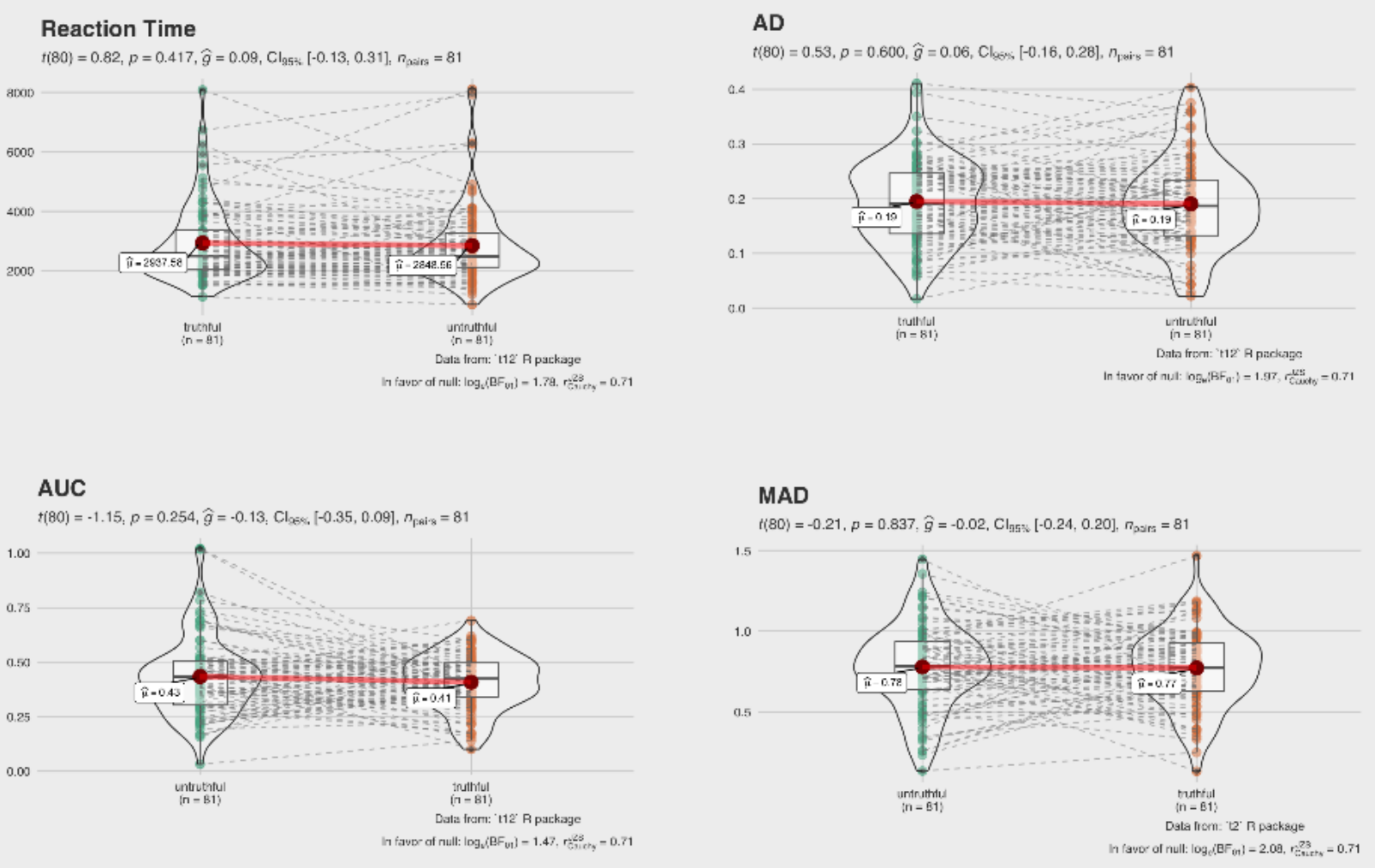

Figure S1 Comparison between behavioral indices of truthful and untruthful responses in experiment 1 